\title{
Efficacy of a new balloon catheter for internal cardioversion of chronic atrial fibrillation without anaesthesia
}

\author{
E Alt, R Ammer, G Lehmann, C Schmitt, J Pasquantonio, A Schömig
}

\begin{abstract}
Objective-To compare a new internal cardioversion system incorporated into a balloon guided catheter with a conventional two electrode system in patients with atrial fibrillation (AF). Design-Prospective study.

Patients-74 patients with chronic AF treated by internal cardioversion.

Materials-A 7.5 F balloon catheter with high energy electrode arrays each consisting of six $0.5 \mathrm{~cm}$ platinum rings. Brachial vein access enables one electrode array to be placed in the left pulmonary artery (distal pole) and the other at the lateral right atrial wall (proximal pole). The conventional two electrode system consists of $6 \mathrm{~F}$ electrodes placed in the proximal left pulmonary artery (anode) and the lower right atrium.

Interventions-Internal cardioversion was performed by shocks delivered in $40 \mathrm{~V}$ incremental steps from an external defibrillator. Shocks were applied by the new device to 32 patients (group $A$ ) and by the conventional sysytem to 42 patients (group B).

Results-The groups differed with respect to system positioning $(9.2(7.3) v 12.3(8.1)$ minutes, $\mathbf{p}<0.05$ ) and fluoroscopy times (1.7 (1.0) $v 3.3$ (2.1) minutes, p $<0.01)$. Sinus rhythm was restored in 30 patients of group $A$ and in 39 of group B (NS) with mean (SD) energy requirements of 8.4 (3.1) J and 7.2 (3.1) J, respectively (NS). Conclusions-This new method of internal cardioversion has comparably high primary success rates and low sedation requirements with single and two lead systems.
\end{abstract}

(Heart 1998;79:128-132)

Keywords: atrial fibrillation; catheter; defibrillation; internal cardioversion

Atrial fibrillation (AF) is one of the most common chronic cardiac arrhythmias. The prevalence of this condition increases with age and concomitant heart disease. ${ }^{1-4}$ The risk of thromboembolic events in patients with $\mathrm{AF}$ is about six times greater than in patients with sinus rhythm ${ }^{5}$; stroke volume and cardiac output are also diminished by up to $35 \%,{ }^{67}$ and cardiopulmonary exercise capacity is reduced, especially in the presence of underlying heart disease. ${ }^{8}$ Thus, in principle the preservation of sinus rhythm is worthwhile.
Apart from pharmacological approaches leading to primary success rates of $40-70 \%,{ }^{6-11}$ electrical external cardioversion necessitates deep sedation and has a reduced efficacy in patients with a high body mass index. ${ }^{12} 13$

A new method of internal cardioversion for restoration of sinus rhythm using optimised electrode positions that encompass as much atrial tissue as possible ${ }^{14}{ }^{15}$ has been reported in animals $^{141617}$ and $\operatorname{man}^{13151819}$ and is superior to conventional external cardioversion in terms of primary success rates, energy requirements, and the need for sedation. Present data have been collected using two part internal devices, which despite their advantages compared with those of external cardioversion also have some disadvantages, such as lack of ventricular back up stimulation or prolonged fluoroscopy times. ${ }^{141617}$

A new catheter has been devised to enable development of an optimal electrical field, to allow pacing and sensing of atrial and ventricular tissue and monitoring of haemodynamic variables. An important aim was ease of handling, keeping fluoroscopy times low and yielding similar primary success rates to those with conventional two part electrodes at comparable energy levels. ${ }^{20}$ Cardioversion with this catheter is compared with that achieved with a conventional two part electrode system in patients with chronic AF.

Patients and methods

PATIENT CHARACTERISTICS

Patients aged 21 to 75 years were enrolled if the following criteria were fulfilled: chronic AF of at least 14 days documented by serial electrocardiogram (ECG); effective anticoagulation with warfarin for at least two weeks (international normalised ratio (INR) 2.5-4.2); and absence of left atrial or atrial appendage thrombus (assessed by transoesophageal echocardiography immediately before cardioversion to preclude any thromboembolic risk).

Patients were excluded when there was evidence of digitalis toxicity, abnormal electrolyte concentrations, or hyperthyroidism. Furthermore, patients with a history of long QT syndrome or acute myocardial infarction within the past six weeks, or of embolism were excluded. Clinical examination was performed and a medical history taken. All patients underwent 12-lead electrocardiography, 24 hour Holter monitoring, chest radiography, and $M$ mode (also for determination of left atrial size) and Doppler echocardiography. 
Table 1 Characteristics of patients with chronic atrial fibrillation undergoing internal cardioversion attempts using a pulmonary artery/right atrium vector with two different catheter system

\begin{tabular}{|c|c|c|}
\hline & \multicolumn{2}{|c|}{ Internal cardioversion } \\
\hline & $\begin{array}{l}\text { Balloon catheter } \\
\text { (group } A)\end{array}$ & $\begin{array}{l}\text { Two catheter } \\
\text { system (group B) }\end{array}$ \\
\hline Number of patients & 32 & 42 \\
\hline Age & $60(10)$ & $57(8)$ \\
\hline Male/female ratio & $26 / 6$ & $33 / 9$ \\
\hline Body weight (kg) & $75(13)$ & $78(14)$ \\
\hline Height $(\mathrm{cm})$ & $173(11)$ & $176(10)$ \\
\hline Left atrial size $(\mathrm{mm})$ & $59(6)$ & $61(6)$ \\
\hline \multicolumn{3}{|l|}{ Underlying heart disease } \\
\hline Coronary artery disease & 7 & 9 \\
\hline Hypertension & 9 & 9 \\
\hline Valvar & 6 & 8 \\
\hline Dilated cardiomyopathy & 4 & 7 \\
\hline Hypertrophic cardiomyopathy & 0 & 1 \\
\hline Lone atrial fibrillation & 5 & 7 \\
\hline Miscellaneous & 1 & 1 \\
\hline Duration of current episode of $\mathrm{AF}$ (months) & $10.6(6.2)$ & $9.7(7.5)$ \\
\hline Range (months) & $0.5-16$ & $0.5-34$ \\
\hline $\begin{array}{l}\text { Previous unsuccessful attempts of pharmacological } \\
\text { conversion (number of patients) }\end{array}$ & 19 & 26 \\
\hline
\end{tabular}

Values are mean (SD). No signficant difference between groups for any parameter.

Routine laboratory and thyroid variables were also measured.

A total of 74 consecutive patients with chronic AF of at least two weeks duration (mean (SD) 10.0 (7.3) months, range 0.5-34 months) were evaluated. Informed consent was obtained from all patients after thorough explanation of the benefits and risks of the study. The study was performed according to the protocol approved by the Human Research Ethics Committee of the Klinikum rechts der Isar.

Patients were treated by internal cardioversion using either the newly developed balloon catheter (32 patients, group A), in an attempt to minimise fluoroscopy time and simplify applicability of the device, or a conventional two part system (42 patients, group B) (table 1).

\section{ANTICOAGULATION}

Patients with AF were treated with warfarin sodium for at least three weeks before defibrillation. The dose was adjusted to maintain an INR of 2.5-4.2. Warfarin sodium was withheld 48 hours before cardioversion, and an INR repeated on the same day of less than 3.0 was required to perform the procedure because of the risk of bleeding from puncture sites. Administration of warfarin sodium was resumed after cardioversion and continued for either four weeks in patients who successfully reverted to sinus rhythm or indefinitely in those who remained in AF. The long term INR was adjusted individually according to patient characteristics.

PREREQUISITES AND CONSTRUCTION OF THE NEW CATHETER

The single lead atrial conversion catheter (EP Med Systems, Mount Arlington, New Jersey, USA) is a $7.5 \mathrm{~F}$, latex balloon tipped device for guidance through the heart that resembles a Swan-Ganz catheter (fig 1). The high energy electrode arrays consist of six $0.5 \mathrm{~cm}$ platinum rings with a total active surface area of $2.5 \mathrm{~cm}^{2}$. The two arrays are separated by $18 \mathrm{~cm}$. The catheter is advanced so that one electrode array is positioned in the left pulmonary artery and the other at the lower right atrial wall, the proximal of which forms the cathode (group A). ${ }^{20}{ }^{21}$ Additional single electrode rings are located on the catheter for pacing and sensing of the atrium and ventricle. A central lumen offers the possibilities of guidewire application, blood sampling, pressure monitoring, and drug administration.

CONVENTIONAL TWO LEAD SYSTEM

The reference device for internal cardioversion of chronic AF (group B) was a catheter system consisting of two temporary, custom built $6 \mathrm{~F}$ catheter (Elecath, Rahway, New Jersey, USA) defibrillation electrodes with an active surface area of $2.5 \mathrm{~cm}^{2}$, each consisting of nine parallel stainless steel rings. The cathode catheter was positioned in the lower right atrium and the anodal one in the proximal left pulmonary artery. One separately connected electrode pole was used for sensing and pacing of the atrium (fig 2). ${ }^{20}$

\section{DEFINITIONS}

Chronic AF was defined from the ECG as a narrow QRS complex rhythm without $P$ waves or flutter waves and with an irregular

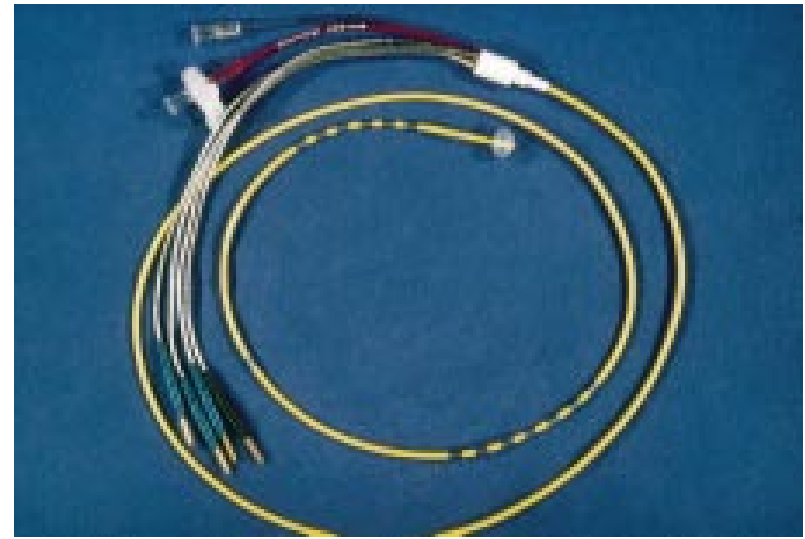

Figure 1 Custom built, single lead Swan-Ganz cardioversion catheter system. The middle of the atrial rings is individually connected for sensing and pacing, while others are connected in parallel (atrial array). One pole in the ventricle serves for ventricular sensing and stimulation, and the pulmonary rings are connected in parallel for atrial defibrillation (pulmonary array).

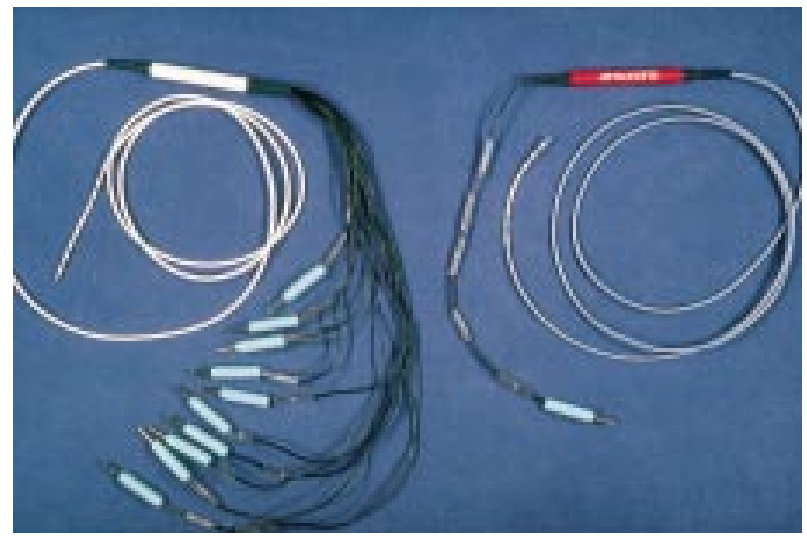

Figure 2 Conventional two part catheter system for internal cardioversion of atrial fibrillation. The electrode on the left has individually connected rings suitable for sensing and stimulation of the atrium through one pole, while the others are used for shock application. All poles are in parallel in the electrode on the right. 

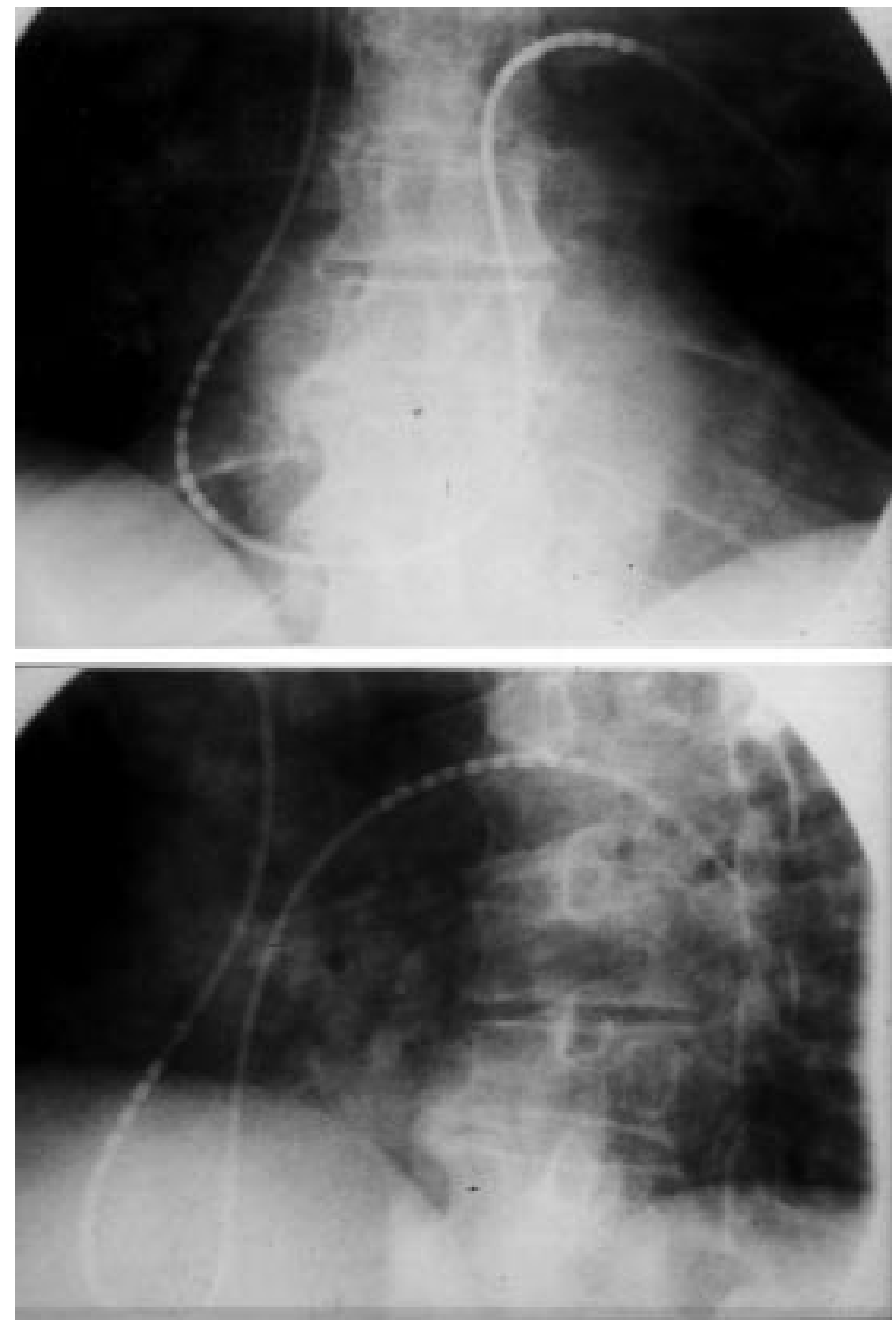

Figure 3 Frontal (top) and left anterior oblique (bottom) chest radiographs of the new balloon catheter device in situ by means of brachial vein access ready for internal cardioversion of atrial fibrillation.

ventricular response of at least two weeks duration. Successful cardioversion was defined as the resumption of sinus rhythm within $30 \mathrm{sec}-$ onds of the shock and persisting for at least one minute.

Table 2 Short and long term results of intracardiac cardioversion attempts in patients with chronic atrial fibrillation using two different catheter systems

\begin{tabular}{|c|c|c|c|}
\hline & \multicolumn{2}{|c|}{ Internal cardioversion } & \multirow[b]{2}{*}{ Significance } \\
\hline & $\begin{array}{l}\text { Balloon catheter } \\
\text { (group } A \text { ) }\end{array}$ & $\begin{array}{l}\text { Two catheter system } \\
\text { (group B) }\end{array}$ & \\
\hline Number of patients & 32 & 42 & NS \\
\hline Defibrillation threshold $(\mathrm{J})$ & $8.4(3.1)$ & $7.2(3.1)$ & NS \\
\hline Voltage (V) & $368(139)$ & $355(127)$ & NS \\
\hline Impedance $(\Omega)$ & $53.5(5.1)$ & $54.8(8.4)$ & NS \\
\hline Acute outcome & $30 / 32 \mathrm{SR}$ & $39 / 42 \mathrm{SR}$ & NS \\
\hline Complications & None & 1 Haematoma & \\
\hline Catheter placement time (min) & $9.2(7.3)$ & $12.3(8.1)$ & $\mathrm{p}<0.05$ \\
\hline Fluoroscopy time (min) & $1.7(1.0)$ & $3.3(2.1)$ & $\mathrm{p}<0.01$ \\
\hline $\begin{array}{l}\text { Interventional stimulation for bradycardia } \\
\text { (number of patients) }\end{array}$ & $6 / 32$ & $9 / 42$ & NS \\
\hline Duration of follow up (months) & $11.1(4.2)$ & $11.4(5.3)$ & NS \\
\hline Intention to treat long term outcome & SR $17 / 30$ & SR $19 / 39$ & NS \\
\hline
\end{tabular}

Values are mean $(\mathrm{SD})$

$\mathrm{SR}$, sinus rhythm.
PROTOCOL FOR INTERNAL CONVERSION

Internal cardioversion was performed in the cardiac catheterisation laboratory. The patients were sedated with $5 \mathrm{mg}$ diazepam given orally. An additional 2-12 mg midazolam was administered intravenously before cardioversion if requested by the patient.

In group $A$, the balloon guided catheter was advanced by brachial vein access (fig 3), not least for reasons of facilitated haemostasis after cardioversion in anticoagulated patients as compared with that when a femoral approach is used (table 2).

In group B, the two lead system catheter was inserted through the right femoral vein and placed in the lower right atrium and in the proximal pulmonary artery (fig 4 ).

In each group, placement time was measured as the time period starting with puncture of the respective vein and ending with connection of the electrode system in situ to the defibrillator immediately before cardioversion. Fluoroscopy time was measured by the radiation device itself during placement of the respective electrode system.

Biphasic shocks of $3 \mathrm{~ms} / 3 \mathrm{~ms}$ pulse duration with phases separated by $0.2 \mathrm{~ms}$ were used for internal cardioversion. The shocks were delivered by an external defibrillator (Ventritex HVS-02, Ventritex, Sunnyvale, California, USA) and synchronised to the $\mathrm{R}$ wave with a minimum $\mathrm{R}-\mathrm{R}$ interval of $500 \mathrm{~ms}$. Starting with a test shock of $60 \mathrm{~V}$ intensity, the energy was increased in $40 \mathrm{~V}$ steps until either cardioversion or a maximum of $520 \mathrm{~V}$, corresponding to about $12 \mathrm{~J}$, was achieved. Criteria for discontinuation were patient discomfort, complications, such as induction of proarrhythmia or major bleeding, and shock energies above $12 \mathrm{~J}$.

The shock waveform was digitised at $100 \mathrm{kHz}$ and processed to evaluate waveform morphology, delivered voltage, current, energy, and electrode impedance using a customised Macintosh computer. Impedance and energy were calculated by a specially written LabVIEW software program (National Instruments, Austin, Texas, USA).

FOLLOW UP

All patients were followed up in our outpatient department. A 12-lead ECG was obtained at one week, one month, and three to 12 months or earlier if there were symptoms suggestive of recurrent AF. All patients were treated with sotalol with at least $80 \mathrm{mg}$ twice daily (mean daily dose 174 (54) $\mathrm{mg}$, range 160-400) after effective conversion to sinus rhythm. Additionally, angiotension converting enzyme inhibitors, diuretics, and digitalis were administered according to the patient's clinical status. Anticoagulation was discontinued after four weeks in patients with persistent sinus rhythm.

STATISTICAL ANALYSIS

Continuous variables are expressed as means (SD). Statistical univariate and multivariate analyses were performed using the MannWhitney U test or Student's $t$ test/analysis of variance as appropriate by statistical package 

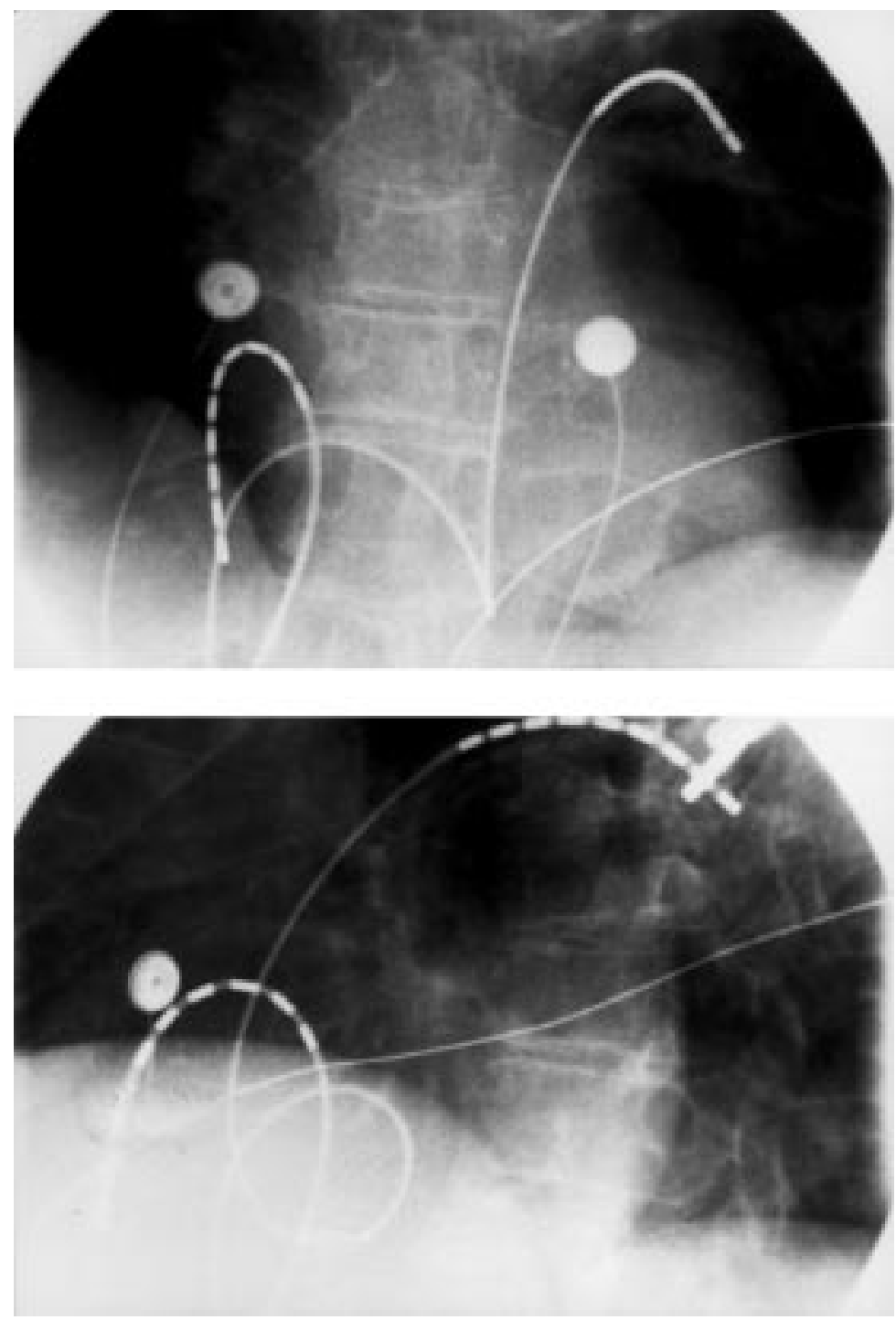

Figure 4 Frontal (top) and left anterior oblique (bottom) chest radiographs of the conventional two part system in situ. One electrode is positioned in the lower right atrium and the second in the left pulmonary artery by means of femoral vein access.

for social sciences (SPSS Inc, Chicago, Illinois, USA) with respect to influence of clinical variables, outcome, shock variables, and follow up. A $p$ value of $<0.05$ was considered significant.

\section{Results}

PATIENT CHARACTERISTICS AND GENERAL RESULTS

There were no significant differences between the two internal cardioversion method groups (table 1). Maximum voltage was responsbile for treatment being stopped in patients with unsuccessful cardioversion. Despite only moderate sedation during internal cardioversion no trial was stopped because of patient discomfort.

EASE OF ELECTRODE SYSTEM PLACEMENT Catheter placement time was significantly shorter in group A (9.2 (7.3) minutes) than in group B (12.3 (8.1) minutes, $\mathrm{p}<0.05)$. Fluoroscopy time was also less in group A (1.7 (1.0) minutes) than in group B (3.3 (2.1) minutes, $\mathrm{p}<0.01$ ) (table 2).
ENERGY REQUIREMENTS AND ACUTE EFFICACY OF CARDIOVERSION

Table 2 presents the results from the one part and two part internal cardioversion groups. The mean energy for successful cardioversion was 8.4 (3.1) J in group A and 7.2 (3.1) J in group B (two part catheter system; NS). Both treatments were comparable with respect to primary success rates $(30 / 32(94 \%)$ in group A $v 39 / 42(93 \%)$ in group B, NS).

LONG TERM CLINICAL OUTCOME

On an intention to treat basis, 17 of 30 patients in group A $(57 \%)$ and 19 of 39 patients in group B $(49 \%$, NS) remained in sinus rhythm during a mean (SD) follow up of 6.6 (2.9) months (range 1-34) (table 2).

COMPLICATIONS

There were no proarrhythmic effects or thromboembolic complications after delivery of the synchronised shocks in either group. One clinically relevant haematoma occurred after intervention in group B (table 2). There were no meaningful increases in creatine kinase activities either before or six hours after cardioversion (53v63 U/1, NS).

\section{Discussion}

CATHETER POSITIONING AND FLUOROSCOPY TIMES

The major finding of this study comparing two different internal cardioversion devices is the ease of placement of the new balloon catheter system, reducing placement time by $25 \%$ and fluoroscopy time by $48 \%$. This fact not only keeps radiation of patients and personnel low, but also, at least theoretically, facilitates placement by the aid of pressure curves readable from the monitor - that is, in settings where rapid cardioversion is mandatory without a fluoroscopy device. As this new electrode system is similar to a conventional Swan-Ganz balloon catheter offering additional facilities of haemodynamic monitoring, intravenous drug application, and transient cardiac sensing and pacing simultaneously, these qualities render it well suited for application in the electrophysiology laboratory as well as in emergency or intensive care settings. ${ }^{22}$ More than $30 \%$ of patients undergoing cardiovascular surgery develop intermittent $\mathrm{AF}$ or flutter perioperatively. ${ }^{23}$ In this setting, the new electrode system would offer a means of rapidly cardioverting AF without the assistance of an anaesthetist; as it would when cardiologists want to perform cardioversion of AF in outpatient departments.

SIDE EFFECTS, TOLERABILITY, AND LONG TERM RESULTS

There were no differences in energy requirements, primary success rates, or procedure related symptomatic effects between the two groups. Moreover, preinterventional sedation was sufficient in all patients and none of the cardioversions had to be interrupted because of patient discomfort. Furthermore, an increase in creatine kinase activities was not detected, nor was there any meaningful difference 
regarding relapse rate into $\mathrm{AF}$ in the long term. Finally, clinically relevant proarrhythmic effects or conduction disturbances attributable to electrode positions or shock energies were not seen with either system, ${ }^{14}{ }^{17}$ such that, in summary, both internal cardioversion modalities can be regarded as comparably safe.

CONCLUSIONS

This newly devised catheter for internal cardioversion of $\mathrm{AF}$ is equally effective as a conventional two part system in terms of primary success rates, energy requirements, and periprocedural sedation. There were no adverse events with either system. Advantages of the new balloon are its ease of placement, low fluoroscopy times, and the ability to perform cardioversions by cardiologists without the assistance of another group of doctors. In addition, as the electrodes are fitted to a modified Swan-Ganz catheter, the new system can be used to perform additional functions-for example, drug administration, blood sampling, monitoring of haemodynamic variables, catheter manoeuvres requiring the use of a guidewire, and stimulating and recording of both atrial and ventricular signals, the latter even during electrophysiological examination. ${ }^{22}$

The authors are grateful to Martin Coenen, MD, Marty Combs, BSE, Maria Montero, MD, and Parwis Fotuhi, MD, for their valuable assistance in conducting the study.

1 Diker E, Aydogdu S, Özdemir M, et al. Prevalence and predictors of atrial fibrillation in rheumatic valvular heart disdictors of atrial fibrillation in rhe

2 Kannel WB, Abbott RD, Savage DD, et al. Epidemiologic features of chronic atrial fibrillation: the Framingham study. N Engl F Med 1982;306:1018-22.

3 Sakamoto H, Okamoto E, Imataka K, et al. Prediction of early development of chronic nonrheumatic atrial fibrillation. fpn Heart f 1995;36:191-9.

4 Sgarbossa EB, Pinski SL, Maloney JD, et al. Chronic atrial fibrillation and stroke in paced patients with sick sinus syndrome. Relevance of clinical characteristics and pacing modalities. Circulation 1993;88:1045-53.

5 Halperin JL, Rothlauf EB. Stroke prevention in atrial fibrillation. Mt Sinai 7 Med 1993;60:289-94.

6 Juul-Möller S, Edvardsson N, Rehnqvist-Ahlberg N. Sotalol versus quinidine for the maintenance of sinus rhythm after direct current conversion of atrial fibrillation. Circulation 1990;82:1932-9.

7 Ruskin J, McHale PA, Harley A, et al. Pressure-flow studies in man: effects of atrial systole on left ventricular function. f Clin Invest 1970:49:472-8.

8 Ueshima K, Myers J, Ribisl PM, et al. Hemodynamic determinants of exercise capacity in chronic atrial fibrillation. Am Heart f 1993;125:1301-5.

9 Coplen SE, Antman EM, Berkis JA, et al. Efficacy and safety of quinidine therapy for maintenance of sinus rhythm after lation 1990;82:1106-116.

10 Skoularigis J, Röthlisberger C, Skudicky D, et al. Effectiveness of amiodarone and electrical cardioversion for chronic rheumatic atrial fibrillation after mitral valve surgery. $A m \mathcal{f}$ Cardiol 1993;72:423-7.

11 Brembilla-Perrot B. Predictive factors of recurrent atrial fibrillation following restoration of sinus rhythm. Editorials in Cardiology 1995;1:56-60.

12 Lown B, Amarasingham R, Neuman J. New method for terminating cardiac arrhythmias: use of synchronized capacitor discharge. $\mathcal{F} A M A$ 1962;182:548.

13 Schmitt C, Alt E, Plewan A, et al. Low-energy intracardiac cardioversion after failed conventional external cardioversion of atrial fibrillation. 7 Am Coll Cardiol 1996;28:994-9.

14 Cooper RAS, Alferness CA, Smith WM, et al. Internal cardioversion of atrial fibrillation in sheep. Circulation dioversion of atrial

15 Alt E, Schmitt C, Ammer R, et al. Initial experience with intracardiac atrial defibrillation in patients with chronic atrial fibrillation. PACE 1994;17:1067-78

16 Powell AC, Garan H, McGovern BA, et al. Low energy conversion of atrial fibrillation in the sheep. $7 \mathrm{Am}$ Coll Cardiol 1992;20:707-11.

17 Kumagai K, Yananouchi Y, Tashiro N, Hiroki T, Arakawa $\mathrm{K}$. Low energy synchronous transcatheter cardioversion of atrial flutter/fibrillation in the dog. $7 \mathrm{Am}$ Coll Cardiol 1990; 16:497-501.

18 Alt E, Ammer R, Lehmann G, et al. Patient characteristics and underlying heart disease as predictors of recurrent and underlying heart disease as predictors of recurrent atrial fibrillation following internal
sion. Am Heart f 1997;134:419-25.

19 Lévy S, Lauribe P, Dolla E, et al. A randomized comparison of external and internal cardioversion of chronic atrial fibrillation. Circulation 1992;86:1415-20.

20 Alt E, Schmitt C, Ammer R, et al. Effect of electrode position on outcome of low-energy intracardiac cardioversion for treatment of atrial fibrillation. Am F Cardiol 1997; 79:621-5.

21 Ideker RE, Wolf PD, Alferness CA, et al. Current concepts for selecting the location, size, and shape of defibrillation electrodes. PACE 1991;14:227-40.

22 Schmitt C, Schneider M, Alt E, et al. Intraatrial defibrillation for cardioversion of atrial fibrillation during radiofrequency ablation in a patient with WPW syndrome. Herzschr quency ablation in a patient
Elektrophys 1997;8:72-5.

23 Waldo AL. Atrial fibrillation following open heart surgery: mechanism and treatment. In: Olsson SB, Allessie MA, Campbell RWF, eds. Atrial fibrillation: mechanisms and therapeutic strategies. Armonk: Futura Publishing, 1994: 211-23.

24 Ortiz J, Sokoloski MC, Niwano S, et al. Successful atrial defibrillation using temporary pericardial electrodes [abstract]. F Am Coll Cardiol 1994;23:125. 